\title{
Marizomib activity as a single agent in malignant gliomas: ability to cross the blood-brain barrier
}

\author{
Kaijun Di, G. Kenneth Lloyd, Vivek Abraham, Ann MacLaren, Francis J. Burrows, Annick Desjardins, \\ Mohit Trikha, and Daniela A. Bota
}

University of California, Irvine, California (K.D., V.A., D.A.B.); Triphase Accelerator Corporation, San Diego, California (G.K.L., A.M., F.J.B, M.T.); The Preston Robert Tisch Brain Tumor Center, Duke University Medical Center, Durham, North Carolina (A.D.)

Corresponding Author: Daniela A. Bota, MD, PhD, 200 S. Manchester Ave., Suite 206, Orange, CA 92868 (dbota@uci.edu).

\begin{abstract}
Background. The proteasome plays a vital role in the physiology of glioblastoma (GBM), and proteasome inhibition can be used as a strategy for treating GBM. Marizomib is a second-generation, irreversible proteasome inhibitor with a more lipophilic structure that suggests the potential for penetrating the blood-brain barrier. While bortezomib and carfilzomib, the 2 proteasome inhibitors approved for treatment of multiple myeloma, have little activity against malignant gliomas in vivo, marizomib could be a novel therapeutic strategy for primary brain tumors.
\end{abstract}

Methods. The in-vitro antitumor activity of marizomib was studied in glioma cell lines U-251 and D-54. The ability of marizomib to cross the blood-brain barrier and regulate proteasome activities was evaluated in cynomolgus monkeys and rats. The antitumor effect of marizomib in vivo was tested in an orthotopic xenograft model of human GBM.

Results. Marizomib inhibited the proteasome activity, proliferation, and invasion of glioma cells. Meanwhile, free radical production and apoptosis induced by marizomib could be blocked by antioxidant $\mathrm{N}$-acetyl cysteine. In animal studies, marizomib distributed into the brain at $30 \%$ of blood levels in rats and significantly inhibited (>30\%) baseline chymotrypsin-like proteasome activity in brain tissue of monkeys. Encouragingly, the immunocompromised mice, intracranially implanted with glioma xenografts, survived significantly longer than the control animals $(P<.05)$ when treated with marizomib.

Conclusions. These preclinical studies demonstrated that marizomib can cross the blood-brain barrier and inhibit proteasome activity in rodent and nonhuman primate brain and elicit a significant antitumor effect in a rodent intracranial model of malignant glioma.

Keywords: blood-brain barrier, chymotrypsin-like, marizomib, malignant glioma, proteasome inhibition.

Glioblastoma (GBM) is the most common primary neoplasm of the brain and affects 11000 people every year in the United States. ${ }^{1}$ It is a very aggressive tumor, with historical median survival rates $<1$ year if left untreated, and is highly resistant to conventional chemotherapy. ${ }^{2}$ The existing chemotherapeutic treatments for such tumors are based on drugs aimed at inducing extensive DNA damage and disrupting the mitotic machinery of the cells. ${ }^{2,3}$ However, the fact is that malignant gliomas tend to develop resistance to these drugs. ${ }^{4}$ Another major drawback is that these current chemotherapeutic agents affect not only the malignant cells but also the normal neural tissues, generating long-standing neurotoxicity in cancer survivors. ${ }^{5}$ Malignant gliomas are characterized by cellular heterogeneity, and attention has to be paid to the selective tailoring of treatment approaches toward differing neoplastic cell subpopulations. Glioma stem-like cells (GSCs) potentially drive tumor initiation and renewal. ${ }^{6}$ GSCs are resistant to radiation ${ }^{7}$ and possibly resistant to chemotherapy. ${ }^{8}$
The proteasome is a key regulator of normal cell functioning. It is responsible for the degradation of intracellular proteins, which can therefore prevent the accumulation of denatured, misfolded, and/or aged proteins. In normal cells, this accumulation of dysfunctional proteins leads to apoptosis. ${ }^{9,10}$ However, cancer cells evade normal apoptotic pathways via alteration of the proteasomal pathways. ${ }^{11,12}$ Preclinical studies have demonstrated that cancer cells are potentially more vulnerable to proteasome inhibition in comparison with normal cells. ${ }^{13}$ Thus, apoptosis may be selectively induced in cancer cells by inhibiting their proteasome activity. ${ }^{9,14,15}$

The effort to develop new chemotherapeutic drugs based on these ideas led to the discovery of bortezomib (PS-341, Velcade), the first proteasome inhibitor approved by the FDA for the treatment of multiple myeloma and subsequently mantle cell lymphoma. ${ }^{16-18} \mathrm{~A}$ second irreversible proteasome inhibitor, carfilzomib, with a limited 205 proteasome inhibition profile (ie, only the chymotrypsin-like (T-L) proteolytic site was also approved by 
the FDA for the treatment of multiple myeloma (FDA, July 20, 2012). Although effective in the treatment of extracranial cancers, the efficacy of carfilzomib and bortezomib have been limited by their inability to cross the blood-brain barrier (BBB) at efficacious doses; ${ }^{19}$ therefore, they may have only modest activity as treatment for malignant gliomas in vivo. ${ }^{20,21}$

Marizomib (NPI-0052, salinosporamide A) is a secondgeneration irreversible proteasome inhibitor whose bicyclic $\beta$-lactone $\gamma$-lactam structure differs significantly from other peptide-based proteasome inhibitors such as bortezomib and carfilzomib. Marizomib has a broader inhibition profile for the $20 \mathrm{~S}$ proteasome compared with bortezomib and carfilzomib and has been shown to inhibit the CT-L ( $\beta 5$ ) CT-T-laspase-like $(C-L, \beta 1)$ and trypsin-like ( $T$ - L, $\beta 2$ ) activities of the $20 \mathrm{~S}$ proteasome. Moreover, marizomib activates a variety of the caspases (ie, 3,8 , and 9), builds up reactive oxygen species (ROS), and thus induces apoptosis. 22,23

The aim of our study was to determine the effect of marizomib on malignant glioma cells, investigate the ability of marizomib to cross the BBB, and test the antitumor function of marizomib in orthotropic xenograft implantation of human glioma cells in immunocompromised mice. To date, the activity of marizomib in malignant glioma models has been studied only in a few permanent, homogenous, serum-raised glioma cell lines. ${ }^{24}$ Therefore, another purpose of our study was to compare the different responses among GSCs derived from human primary brain tumors, normal neural stem cells (NSCs), and stable malignant glioma cell lines with marizomib.

\section{Materials and Methods}

\section{Chemicals and Reagents}

All standard chemicals, buffers, and reagents, unless otherwise indicated, were purchased from Sigma Aldrich. Marizomib was provided by Triphase Accelerator Corporation and was diluted in $2 \%$ dimethylsulfoxide (DMSO) or $5 \%$ Solutol aqueous solution and used within 2 hours of preparation.

\section{Cell Lines}

The primary brain tumor stem-like cells (low-grade glioma LG-GSC-DB29, LG-GSC-DB30; high-grade glioma HG-GSC-DB17, HG-GSC-DB26, HG-GSC-DB32, HG-GSC-DB33 HG-GSC-HuTuP01; Meningioma-SC-M1, Meningioma-SC-M2), and NSCs (SC23, SC27, and DB31) were isolated from patients as previously described. ${ }^{25-27}$ HuTuP01 cells were a gift from Dr. David Panchision. ${ }^{28}$ The detailed culture conditions and verification of stem-like characteristics were previous published by our group $^{5,26}$ and are included in Supplementary Methods. The human glioma cell lines U-251 MG and D-54 MG were maintained in Dulbecco's modified Eagle's medium/F-12 medium containing $292 \mu \mathrm{g} / \mathrm{mL}$ glutamine, 1\% penicillin/streptomycin, and $10 \%$ fetal bovine serum (Omega Scientific, Inc.).

\section{S Proteasome Activity Assay}

The CT-L activity in cells was measured using the 20S Proteasome Activity assay Kit (Chemicon International Inc.). Briefly, cells were treated with $60 \mathrm{nM}$ marizomib for the indicated time points and then collected. The CT-L activity was measured as relative fluorescence units (RFUs). For the in-vivo proteasome profiling studies (see Supplementary Methods), brain tissues from male cynomolgus monkeys were processed as in the previous mouse study, ${ }^{29}$ and samples were evaluated using Proteasome-Glo Assay Kits (Promega).

\section{Cell Viability}

Cells were exposed for 72 hours to various concentrations of marizomib in the presence or absence of $10 \mathrm{mM}$ of $\mathrm{N}$-acetyl cysteine (NAC) as indicated. Cell viability was determined using the Cell Viability kit II (XTT) (Roche Applied Science).

\section{Invasion Assay}

The invasion assay was performed using the BD BioCoat Matrigel Invasion Chamber (BD Biosciences). After an incubation of 24 hours with marizomib (60 nM), invaded cells were fixed and then stained in hematoxylin. Cell counting was facilitated by photographing the entire membrane through the microscope.

\section{Apoptosis Assay}

D-54 cells were treated with either $20 \mathrm{nM}$ or $60 \mathrm{nM}$ of marizomib for 24 hours. The apoptotic cells were measured using the Annexin V: FITC Apoptosis Detection kit (BD Biosciences). Samples were then analyzed by flow cytometry using B.D. Bioflow. Results are expressed as the percentage of untreated cells.

\section{4', 6-diamidino-2'-phenylindole Staining}

U-251 cells were grown on chamber slides and treated with marizomib at $60 \mathrm{nM}$ for 24 hours. Cells were fixed and then stained with $2 \mu \mathrm{g} / \mathrm{mL}$ of 4 ', 6-diamidino-2'-phenylindole (DAPI) for 10 minutes. Images were captured using a fluorescence microscope.

\section{Caspase Assay}

The induction of caspase- 3 in marizomib-treated D-54 cells was measured using the Apopcyto Caspase-3 Fluorometric assay kit (MBL International Corp.). D-54 cells were grown to subconfluence, and treated with either $20 \mathrm{nM}$ or $60 \mathrm{nM}$ marizomib for 24 hours. A parallel set was also treated with $10 \mathrm{mM}$ NAC to quench any ROS that may have been generated. The effect of NAC was expressed as the percentage change from vehicle-treated cells.

\section{Reactive Oxygen Species Measurements}

D-54 cells were treated with marizomib at $60 \mathrm{nM}$ for 12 hours in the presence or absence of $10 \mathrm{mM}$ of NAC. The intracellular ROS were measured using the OxiSelect ROS assay kit (Cell Biolabs, Inc.). The details are included in Supplementary Methods.

\section{Nervous Tissue Distribution of Radioactivity in Sprague-Dawley Rats Administered a Single Dose of $\left[{ }^{3} \mathrm{H}\right]$ - Marizomib}

After a single i.v. administration of $\left[{ }^{3} \mathrm{H}\right]$-marizomib $(0.1 \mathrm{mg} / \mathrm{kg}$, equivalent to $0.6 \mathrm{mg} / \mathrm{m}^{2}$ ) to 11 male Sprague-Dawley rats 
(311-337 g body weight at randomization), the animals (1 rat per time point) were euthanized 2, 10, and 30 minutes and 1,2 , $4,8,24,48,72$, and 120 hours later. Carcasses were frozen, and sections and tissue concentrations of radioactivity were determined by whole body autoradiography.

\section{Marizomib Maximum-tolerated Dose Determination in Athymic BALB/c nu/nu Mice}

Five groups of $6-8$ week old mice ( $n=3$ mice per group) were treated with either control vehicle (2\% DMSO in $5 \%$ Solutol) or different doses of marizomib (150, 200, 250, and $300 \mu \mathrm{g} / \mathrm{kg}$ ) under the University of California, Irvine, Institutional Animal Care and Use Committee (IACUC)-approved protocol. The drug was administered biweekly for 2.5 weeks (on days 1, 4, 8,11 , and 15 ) into the tail vein of the animals, and the animals were monitored for survival. The maximum tolerated dose (MTD) of marizomib was determined to be $200 \mu \mathrm{g} / \mathrm{kg}$.

\section{Intracranial Xenograft Implantation and Marizomib Treatment}

Under the UC Irvine IACUC - approved protocol, D-54 $\left(1 \times 10^{5}\right)$ cells were implanted into the right frontal lobes of athymic BALB/c nu/nu mice. Four days after the implantation, the mice were randomized to 3 groups $(n=6-8)$ and treated with the control vehicle, marizomib, at the MTD $(200 \mu \mathrm{g} / \mathrm{kg})$, and marizomib at the dose below the MTD to minimize potential human toxicity that might not be noticed in a murine model (MTD-1, $150 \mu \mathrm{g} / \mathrm{kg}$ ). The drug or vehicle control was administered biweekly (on days $1,4,8,11,15$, etc.) into the tail vein of the animals. The survival time was calculated from the day marizomib (or vehicle) treatment was started and the day when a mouse showed moribund signs including hemiparesis, obtundation, hunchback, or weight loss of $>20 \%$ from the maximum weight achieved.

\section{Statistical Analysis}

Statistical analyses were performed, and graphs were generated using Prism 4.0 (GraphPad). All values were presented as mean \pm standard error of the mean when at least 3 observations were available. Statistical significance was measured by unpaired $t$ tests or 1-way ANOVA unless otherwise indicated.

\section{Results}

\section{Marizomib Inhibits the Proliferation, Migration, and Invasion of Glioma Cells}

Since marizomib is a second-generation proteasome inhibitor, we first examined its effect on the 205 proteasome activity of stable malignant glioma cell lines U-251 and D-54. The results showed that marizomib had a profound effect on the proteasome activity of both U-251 and D-54 cells; the CT-L activity in both cell types had been reduced to $\sim 15 \%$ after 4 hours of treatment (Fig. 1A). The viability of D-54 and U-251 cells was measured following exposure to increasing concentrations of marizomib. The IC50 was calculated to be $\sim 52 \mathrm{nM}$ for U-251 and $\sim 20 \mathrm{nM}$ for D-54 (Fig. 1B).
It has been reported that GSCs have a lower baseline proteasome activity than the differentiated glioma tumor mass cells, as well as NSCs, ${ }^{5,30}$ and are more sensitive to proteasome pathway inhibition by bortezomib. ${ }^{5}$ We next compared the response to marizomib among low- and high-grade GSCs, meningiomaderived primary cultures, and NSCs. As shown in Fig. 1C, marizomib rapidly and significantly inhibited the CT-L activity in all cell types tested, and malignant GSCs had a lower baseline proteasome activity than NSCs, which was consistent with our previous study. ${ }^{5}$ Meanwhile, marizomib had a differential effect on cell viability: while NSCs, meningiomas, and low-grade GSCs were insensitive to marizomib, high-grade GSCs showed at least $40 \%$ reduction in survival at $20 \mathrm{nM}$ (Fig. 1D) with IC50 ranging from $9.32 \mathrm{nM}-51.06 \mathrm{nM}$ (Supplementary material, Table S1). The similar sensitivity of high-grade GSCs and the stable glioma cultures U-251 and D-54 also suggest that U-251 and D-54 could be considered a good model system for studying the mechanism of action of marizomib in gliomas for the rest of our experiments.

Since the invasive behavior of malignant gliomas contributes to their poor prognosis, ${ }^{31}$ the role of marizomib on cell invasion was measured using Matrigel invasion assay (Fig. 1E). The results showed that the capability of cell invasion was robustly inhibited $(P<.001)$ by marizomib. Wound closure assay (Supplementary material, Fig. S1) further revealed that while the gap was only partially filled in marizomib-treated U-251 cells (even after 30 hours), control cells were able to migrate in and almost fill up the gap within 24 hours.

\section{Marizomib Induces Apoptosis and Caspase-3 Activation in Glioma Cells}

The growth inhibition induced by marizomib was accompanied with apoptosis induction. Marizomib treatment for 24 hours significantly increased the percentage of Annexin $V$ stained cells (Fig. 2A), and DAPI staining (Fig. 2B) further confirmed marizomib-induced apoptosis represented by dramatic changes within the nucleus into dense granular particles (apoptotic bodies, arrow). Caspase-3 activation is an early step in the apoptosis cascade leading to nuclear fragmentation. Marizomib treatment has led to increased activity of caspase-3 in a dose-dependent manner (Fig. 2C). Meanwhile, significant upregulation of cleaved caspase- 3 and poly (ADP-ribose) polymerase (PARP) was detected in marizomib-treated cells (Fig. 2D).

\section{Marizomib Increases ROS Generation, but NAC Quenches ROS Induction, Blocks Caspase-3 Activation, and Improves the Malignant Glioma Cell Survival}

Proteasome inhibition by bortezomib and marizomib has been linked to increased ROS levels in several types of cancer cell lines including lymphomas, leukemias, and breast and prostate cancers. $^{22,32}$ We sought to determine whether marizomib increases oxidative stress in the D-54 cells by measuring the levels of carboxy-H2DCFDA detectable ROS. We treated D-54 cells with $60 \mathrm{nM}$ of marizomib for 12 hours and captured images using light microscopy (Fig. 3A, a and c) and fluorescent microscopy (Fig. 3A, b and d). The ROS-positive cells increased from a basal level of $28.07 \%$ to $50.75 \%$ in the presence of 
A

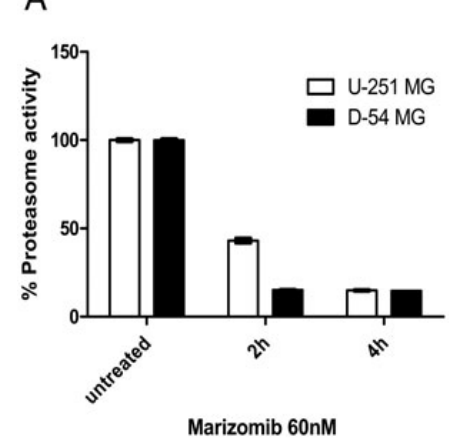

B

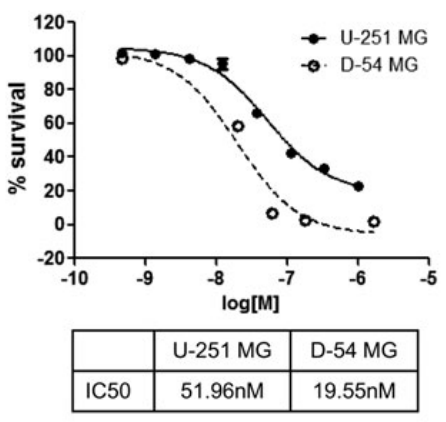

C

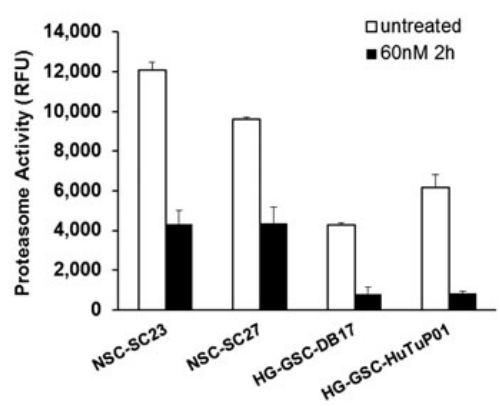

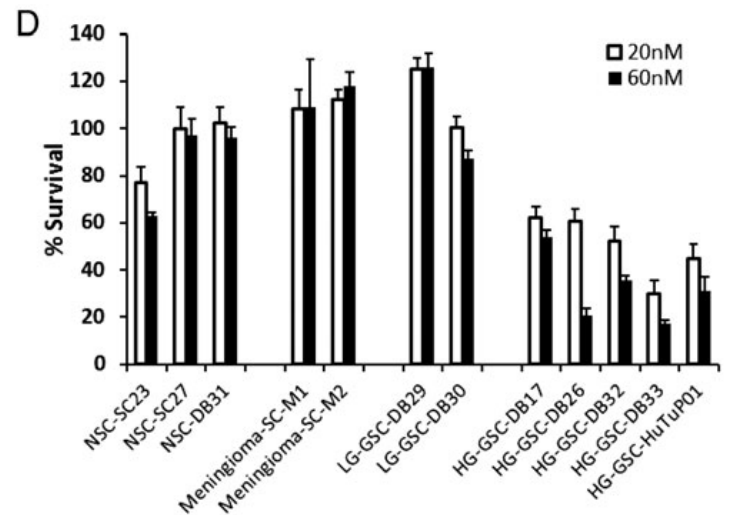

E E D-54 MG U-251 MG

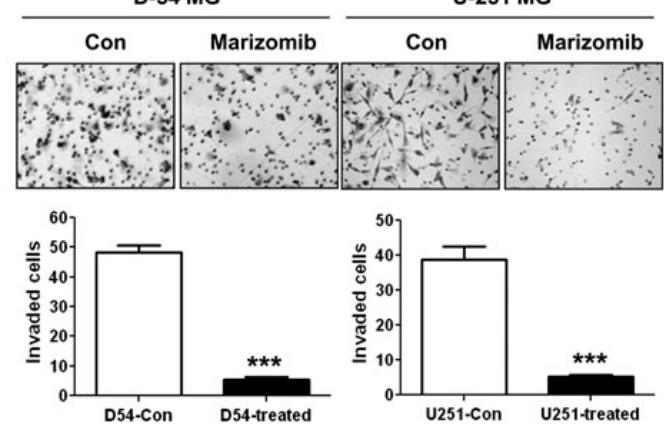

Fig. 1. Marizomib inhibits the proteasome activity, proliferation, and invasion of glioma cells. (A) Proteasome activity was measured in glioma cell lines D-54 and U-251 after treatment with marizomib $(60 \mathrm{nM})$. The chymotrypsin-like activity of treated cells was presented as a percentage of untreated cells. (B) Marizomib treatment for 72 hours effectively reduced survival of D-54 and U-251 cells in a dose-dependent manner. The IC50s were shown in a lower panel. (C) Proteasome activity was measured in neural stem cells and glioblastoma-derived glioma stem cells at baseline and 2 hours after treatment with marizomib $(60 \mathrm{nM})$. (D) Marizomib showed different proliferation inhibition on diverse primary cell cultures after treatment for 72 hours. (E) Invasion capability of cells treated or untreated with $60 \mathrm{nM}$ marizomib for 24 hours was analyzed using Matrigel invasion chambers. The average of invaded cells for each counting grid was shown in the lower panel. ${ }^{* *} P<.001$.

marizomib (Fig. 3A, lower panel). We confirmed this observation by fluorescence spectroscopy, in which case we observed an increase in the ROS-derived fluorescence from $28000 \mathrm{RFU}$ to 40000 RFU under identical conditions (Fig. 3B).

To study the importance of ROS generation as a potential mechanism for marizomib-induced apoptosis, we exposed D-54 cells to the antioxidant NAC (10 mM) starting 1 hour before and continuing throughout the marizomib treatment. As shown in Fig. 3C, the generation of ROS induced by marizomib was completely blocked in the presence of NAC. In addition, NAC dramatically improved D-54 cell survival (Fig. 3D) and abolished caspase-3 activation (Fig. 3E) upon marizomib treatment. Western blot results also revealed that the induction of cleaved caspase- 3 and PARP by marizomib was completely repressed by NAC (Fig. 3F).

\section{Marizomib Crosses the Blood-brain Barrier and Inhibits the Proteasome Activity in Vivo}

Our unpublished studies showed that marizomib behaves similarly with respect to its pharmacokinetic properties in rats and nonhuman primates, with a very short plasma half-life (2-30 min) and a large volume of distribution. Marizomib binds proteasome irreversibly and is subsequently hydrolyzed, which makes the measurement of marizomib in tissue homogenates very challenging. To characterize the pharmacodynamic activity of marizomib in vivo, we evaluated the extent of CNS penetration of marizomib by the quantitative wholebody autoradiography (QWBA) study in Sprague-Dawley rats. After a single i.v. dose of ${ }^{3} \mathrm{H}$-marizomib $(0.1 \mathrm{mg} / \mathrm{kg}$, equivalent to $0.6 \mathrm{mg} / \mathrm{m}^{2}$ ), drug-derived radioactivity was rapidly and widely distributed to tissues in male Sprague-Dawley rats. Maximum blood levels of radioactivity were observed 2 minutes post dose and declined rapidly to a baseline plateau 10 minutes after administration (data not shown). Distribution of radioactivity to the CNS was $\sim 30 \%$ of that for steady-state blood levels (Table 1), demonstrating that marizomib is able to cross the $\mathrm{BBB}$, at least in rodents.

To corroborate the data from rats, a pharmacodynamic experiment was performed in nontumor-bearing cynomolgus monkeys to determine whether marizomib is able to cross the $\mathrm{BBB}$ and inhibit proteasome activity in primate brain. Using Proteasome-Glo assay kits, CT-L, C-L, and T-L activities were assessed in normal monkey prefrontal cortex in vitro, and all 3 proteasome activities were present at levels well distinguished from baseline. (Nonproteasome activity metabolizing the assay substrate was subtracted using duplicate wells in the presence of a supramaximal concentration of proteasome inhibitor) (Fig. 4A). This indicates a wide window for demonstrating differential inhibition of these activities. Next, 


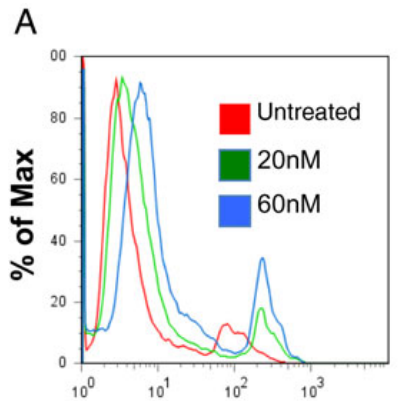

$\mathrm{D}$

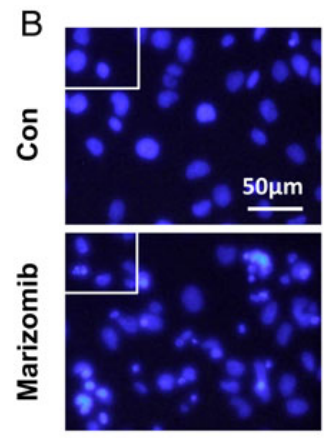

D-54 MG
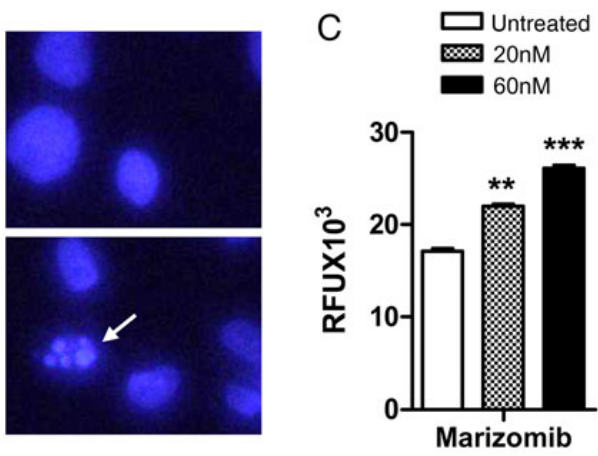

U-251 MG

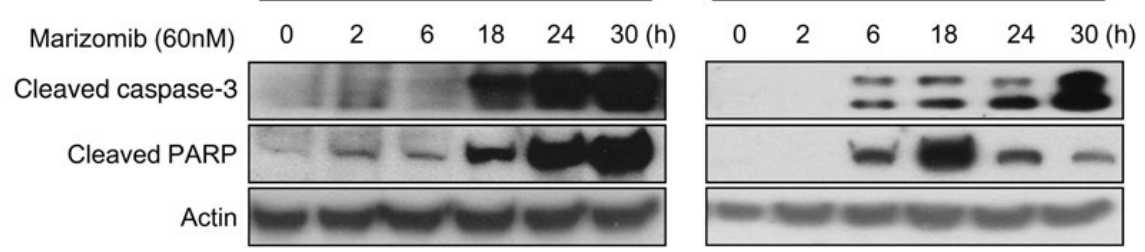

Fig. 2. Marizomib induces apoptosis and caspase-3 activation in glioma cells. (A) FITC Annexin V apoptosis assay kit was used to detect apoptotic cell death induced by marizomib treatment for 24 hours in D-54 cells. (B) DAPI staining was performed to observe apoptotic cells indicated by small, condensed nuclei in D-54 cells treated by marizomib (60 nM) for 24 hours. (C) The caspase-3 activity of D-54 cells was measured using Apopcyto Caspase-3 Fluorometric assay kit 24 hours after treatment with marizomib. An $80 \%$ increase in caspase-3 activity was found in presence of $60 \mathrm{nM}$ marizomib. ${ }^{* *} P<.01$, ${ }^{* *} P<.001$. (D) D-54 and U-251 cells were treated with 60 nM marizomib for indicated time points. Western blot was used to detect cleaved caspase-3 and PARP. Actin was the internal control.

groups of monkeys were treated orally, either once weekly or twice weekl, with marizomib $\left(0.55-0.64 \mathrm{mg} / \mathrm{m}^{2}\right)$, and brain (prefrontal cortex) tissue was harvested 24 hours after the final drug treatment. As shown in Fig. 4B and Supplementary material, Fig. S2, marizomib treatment resulted in a differential inhibition of proteasome activities in monkey prefrontal cortex. CT-L and C-L activities were statistically inhibited to a similar extent (26\%-28\% inhibition, $P<.05)$. Meanwhile, T-L activity was only weakly inhibited (11\%-16\% inhibition). The degree of inhibition observed was very similar for the twice-weekly and once-weekly dosing schedules for all proteasome activities without any statistical differences.

\section{Marizomib Prolongs the Survival of Nude Mice Intracranially Implanted With Human Glioma D-54 MG Xenografts}

We next examined the in-vivo antitumor efficacy of marizomib using human glioma xenografts. The MTD was first determined by injecting nude mice with different doses of marizomib (150, 200,250 , and $300 \mu \mathrm{g} / \mathrm{kg}$, respectively) twice per week for 2.5 weeks. The MTD after 4 treatments was determined to be $200 \mu \mathrm{g} / \mathrm{kg}$, which is equivalent to a human dose of $0.6 \mathrm{mg} / \mathrm{m}^{2}$. There was no statistical difference in the weight of animals between the control, MTD, and MTD-1 (150 $\mu \mathrm{g} / \mathrm{kg})$. MTD-1 is a dose below the MTD and was considered to minimize potential human toxicity that might not be noticed in a murine model (Fig. 5A). We used MTD and MTD-1 for further experiments.

Mice received either vehicle control or marizomib treatment after xenograft implantation. All mice from the control group developed neurological symptoms 15-22 days after the treatment and had to be euthanized (average survival 18 days). The mice treated at the MTD survived significantly longer (average survival 31 days) than the control animals $(P<.05$, log-rank test). Furthermore, $50 \%$ of the mice in the MTD group survived much longer (29-65 days) than any of the control mice, and $25 \%$ of the mice were still alive even after 50 days. However, the survival in the MTD-1 group was not different from that of the control group (Fig. 5B).

\section{Discussion}

Recent studies have demonstrated the effects of the secondgeneration proteasome inhibitor marizomib in multiple myeloma, mantle cell lymphoma, Waldenstrom's macroglobulinemia, and chronic and acute lymphocytic leukemia as well as colorectal and pancreatic cancer models. ${ }^{33}$ However, little is known about its effect on primary brain tumors, particularly in the very aggressive GBM. Our study demonstrated that marizomib displayed antiglioma activity in vitro, suppression of proliferation (Fig. 1B), and inhibition of migration and invasion (Fig. 1E and Supplementary material, Fig. S1) in association with profound inhibition of proteasome activity in $\mathrm{U}-251$ and D-54 cells (Fig. 1A). Proteasome inhibition results in a cascade of events leading to apoptotic cell death. Marizomib treatment led to apoptosis indicated by Annexin-V-positive cells (Fig. 2A) as well as nuclear fragmentation and apoptotic body formation (Fig. 2B). Caspase-3 is a critical executioner of apoptosis as it is either partially or totally responsible for the proteolytic cleavage of many key proteins such as the nuclear enzyme PARP. Our findings that marizomib-induced apoptosis is associated 
A

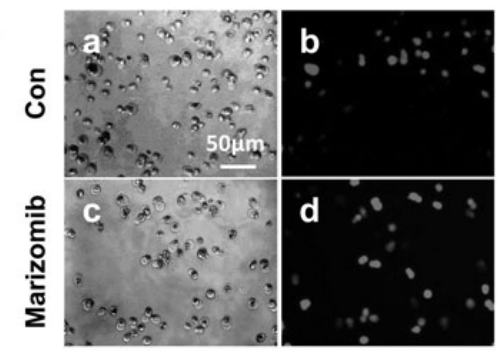

\begin{tabular}{|c|c|}
\hline & $\%$ ROS positive \\
\hline Control & 28.07 \\
\hline $\begin{array}{c}\text { Marizomib } \\
\text { (60nM) }\end{array}$ & 50.75 \\
\hline
\end{tabular}

B

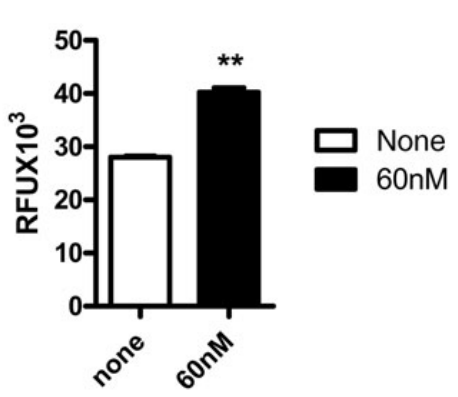

C

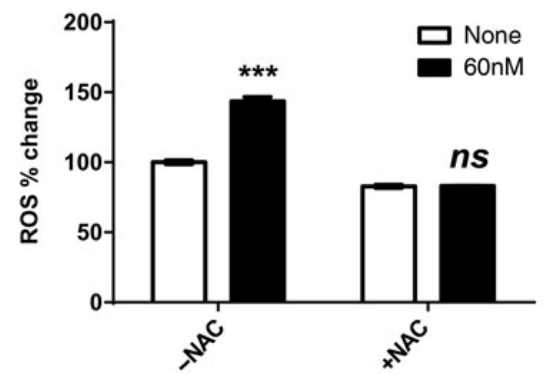

$\mathrm{F}$
D

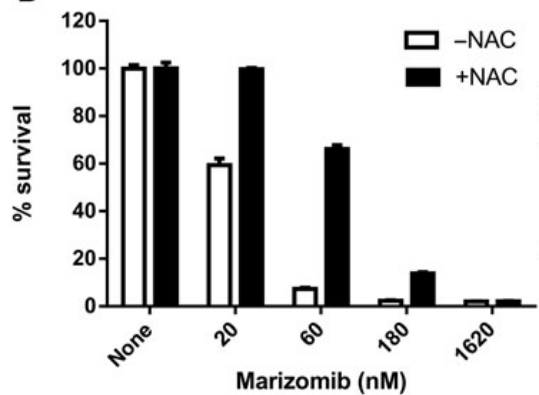

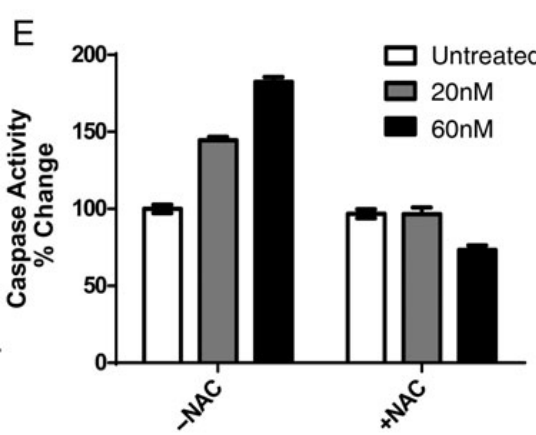

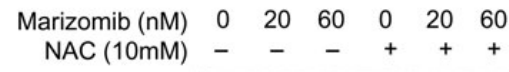

Cleaved Caspase-3

Cleaved PARP

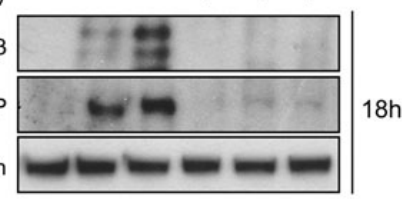

Cleaved Caspase-3

Cleaved PARP

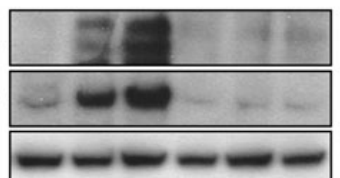

Fig. 3. Marizomib increases reactive oxygen species (ROS) generation, but N-acetyl cysteine (NAC) quenches ROS induction, blocks caspase-3 activation, and improves the survival of D-54 cells. (A) After treatment with marizomib $(60 \mathrm{nM})$ for 12 hours, ROS generation was measured as the number of DCF fluorescent cells by direct fluorescent microscopy. The percentage of ROS-positive cells is shown in the lower panel. (B) Carboxy-H2DCFDA detectable ROS were measured by fluorescence spectroscopy. Values represent the mean \pm SD of 3 experiments. (C) The addition of N-acetyl cysteine (NAC) (10 mM) blocked the marizomib-induced ROS activation. (D) NAC pretreatment was able to rescue the D-54 cells from marizomib-induced cell death. (2-way ANOVA analysis, $P<.0001$ ). (E) The caspase-3 activation triggered by marizomib was abolished by NAC pretreatment (2-way ANOVA analysis, $P<.0001$ ). (F) D-54 cells were treated with 20 or $60 \mathrm{nM}$ marizomib in the presence or absence of $10 \mathrm{mM}$ NAC for indicated time points. Western blot was used to detect cleaved caspase-3 and PARP. Actin was used as the internal control. ${ }^{* *} P<.01$, ${ }^{* * *} P<.001, \mathrm{~ns}=$ not significant.

Table 1. Nervous tissue levels of ${ }^{3} \mathrm{H}$-Marizomib compared with blood in the Sprague-Dawley rats

\begin{tabular}{llll}
\hline Region/Time & $2-30 \mathrm{~min}$ & $1-8 \mathrm{~h}$ & $24 \mathrm{~h}$ \\
\hline Brain: cerebrum & $31.33 \pm 12.77$ & $53.00 \pm 5.70$ & 51.00 \\
Brain: cerebellum & $35.00 \pm 12.86$ & $56.25 \pm 3.04$ & 58.00 \\
Brain: medulla & $24.67 \pm 9.03$ & $38.75 \pm 3.28$ & 40.00 \\
All brain & $30.33 \pm 6.04$ & $49.33 \pm 3.17$ & $49.67 \pm 5.24$ \\
Peripheral nerves & $17.33 \pm 5.81$ & $20.25 \pm 3.64$ & 21.00 \\
Spinal cord & $21.33 \pm 6.94$ & $32.75 \pm 7.23$ & 38.00 \\
Kidney & $320.7 \pm 170.7$ & $250.8 \pm 6.59$ & 178.00 \\
& & & \\
\hline
\end{tabular}

Data expressed as percent blood levels (mean \pm SEM).

with activation of caspase- 3 and cleavage of PARP in glioma (Fig. 2C and D) are similar to previous studies in leukemia. ${ }^{22,23}$

ROS induction as a trigger for apoptosis is a key feature of bortezomib activity in CNS-derived tumor cells. ${ }^{34}$ Marizomib has also been demonstrated to induce ROS in leukemia cells. ${ }^{22}$ We demonstrated here that marizomib could significantly induce ROS production in glioma (Fig. $3 \mathrm{~A}$ and B), and this effect is attenuated in the presence of NAC, a free radical scavenger (Fig. 3C). Furthermore, ROS generation is a major mechanism of action for marizomib-induced apoptosis. Blockage of ROS induction by NAC significantly improved the survival of glioma cells treated with marizomib (Fig. 3D) and diminished the activation of caspase-3 and PARP (Fig. 3E and F).

Our in-vivo study demonstrated that the administration of marizomib in monkeys resulted in significant inhibition of CT-L and C-L activities in brain tissues (Fig. 4 and Supplementary material, Fig. S2). This result is different from a previously published study ${ }^{35}$ in which marizomib administration inhibited 205 proteasome activity in many organs but not in the brain. The difference may be due to a technical artifact. In Singh et al., very low brain proteasome activity was detected at baseline, making the inhibition difficult or impossible to detect. By contrast, baseline proteasome activity levels were much higher in the Williamson study, ${ }^{29}$ and robust inhibition of CT-L proteasome activity was observed in brain as well as blood and tumor. Further evidence for CNS penetration of marizomib has been found in our QWBA study in rats (Table 1). Distribution of radioactivity to the CNS was $30 \%$ that of steady-state blood 

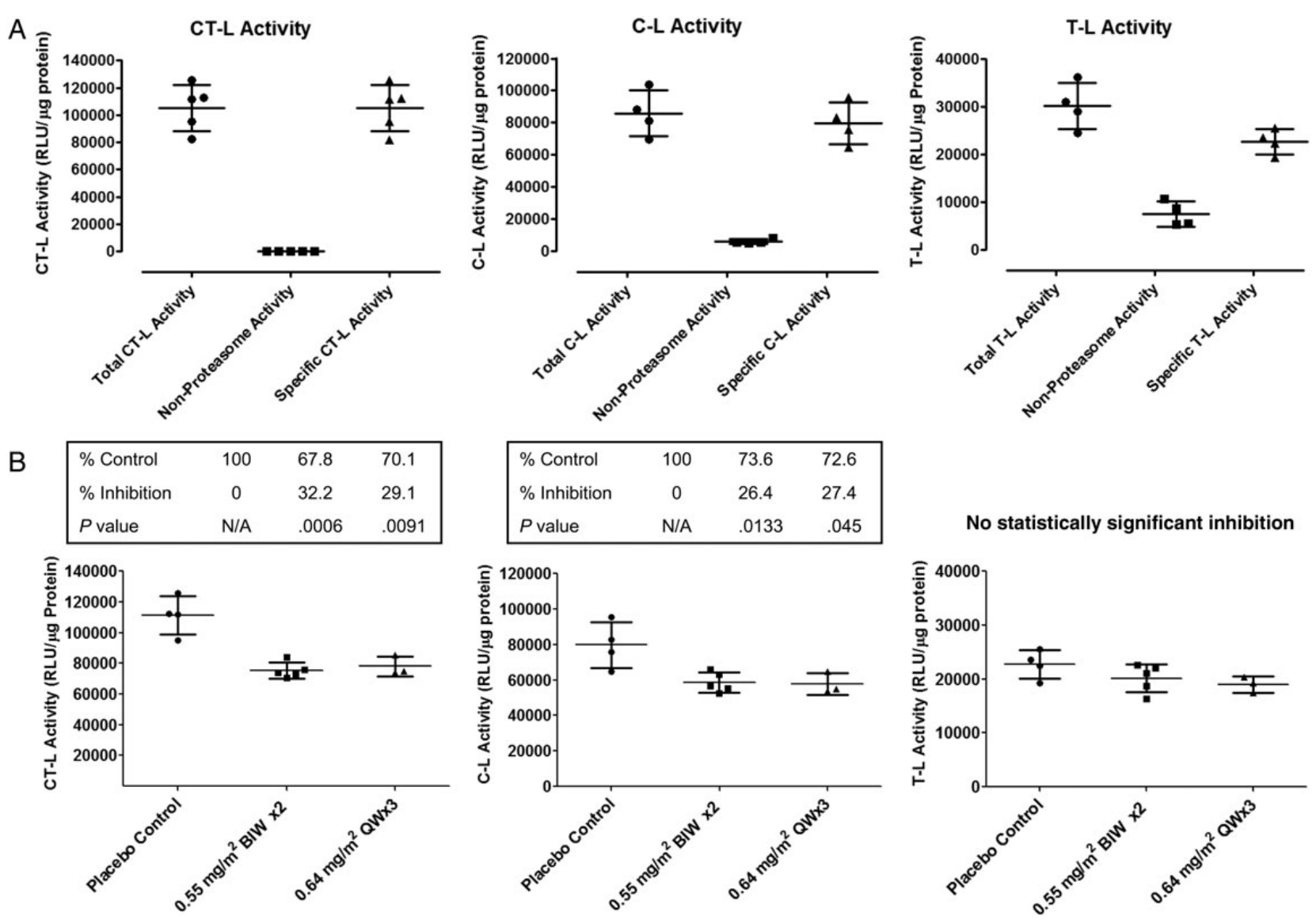

Fig. 4. Effect of marizomib regiments on the proteasome activity of prefrontal cortex in cynomolgus monkeys. (A) The chymotrypsin-like, C-L and T-L activities were assessed in control monkey prefrontal cortex in vivo, and all 3 proteasome activities were present at levels well distinguished from baseline. (B) Marizomib administered orally resulted in a differential inhibition of proteasome activities in monkey prefrontal cortex. The degree of inhibition observed was very similar for the twice-weekly and once-weekly dosing schedules.

levels. Taken together, our findings demonstrate that marizomib is able to cross the BBB and inhibit proteasome activity in rodents and primates. More importantly, marizomib as a single agent may markedly prolong survival of immunodeficient mice intracranially implanted with human glioma xenografts with minimal effect on body weight (Fig. 5).

Another interesting finding from our study was that marizomib demonstrated a remarkable selectivity of growth inhibition for GSCs derived from high-grade tumors compared with GSCS derived from low-grade gliomas, meningiomas, and NSCs (Fig. 1D and Supplementary material, Table S1). Even though marizomib could suppress CT-L activity in both NSCS and GSCs, NSCs have a higher baseline proteasome activity than malignant GSCs (Fig. 1C). These data suggest that marizomib might have limited neurotoxicity. These data confirm our previous publication using another proteasome inhibitor, bortezomib, which also suggested that GSCs are specifically targeted by proteasome inhibition as compared with NSCs. ${ }^{5}$

Recent preclinical data provide compelling evidence to support combining marizomib with other therapies in hard-to-treat solid tumors such as GBM. It has been reported that the combination of marizomib and multiple drugs, (eg, 5-fluorouracil, CPT-11, bevacizumab, oxaliplatin, gemcitabine, and erlotinib) exhibited enhanced antitumor activities in colon and pancreatic carcinoma xenograft models. ${ }^{36,37}$ The current multiple myeloma study (http://www.clinicaltrials.gov, NCT02103335) treats participants with a combination of pomalidomide, marizomib and dexamethasone. Other proteasome inhibitors such as bortezomib can increase the efficacy of both temozolomide ${ }^{38}$ and PI3-kinase inhibitors ${ }^{39}$ in malignant glioma cell models. In addition, marizomib can radiosensitize certain human GBM cells that exhibit significant levels of mutant p53. ${ }^{24}$ The findings in multiple models clearly demonstrate the significant potential of combining marizomib with other cancer-targeted therapies that are currently utilized for the treatment of GBM- and combination therapy with temozolomide or bevacizumab is currently being considered in our laboratory.

We conclude that marizomib is an effective in-vitro agent for targeting the most aggressive and treatment-resistant malignant glioma cells. In addition, marizomib has relatively little effect on NSCs, suggesting the potential for a good neurologic safety profile, which is an important property for a drug that has the ability to cross the BBB. In malignant glioma cells, marizomib inhibits proteasome activity, increases ROS and caspase-3 activation, and induces apoptotic cell death, similar to that reported in hematologic malignancies. These findings correlate the pharmacodynamic effects of marizomib with its cytotoxic effects against glioma in vivo. Marizomib is now 

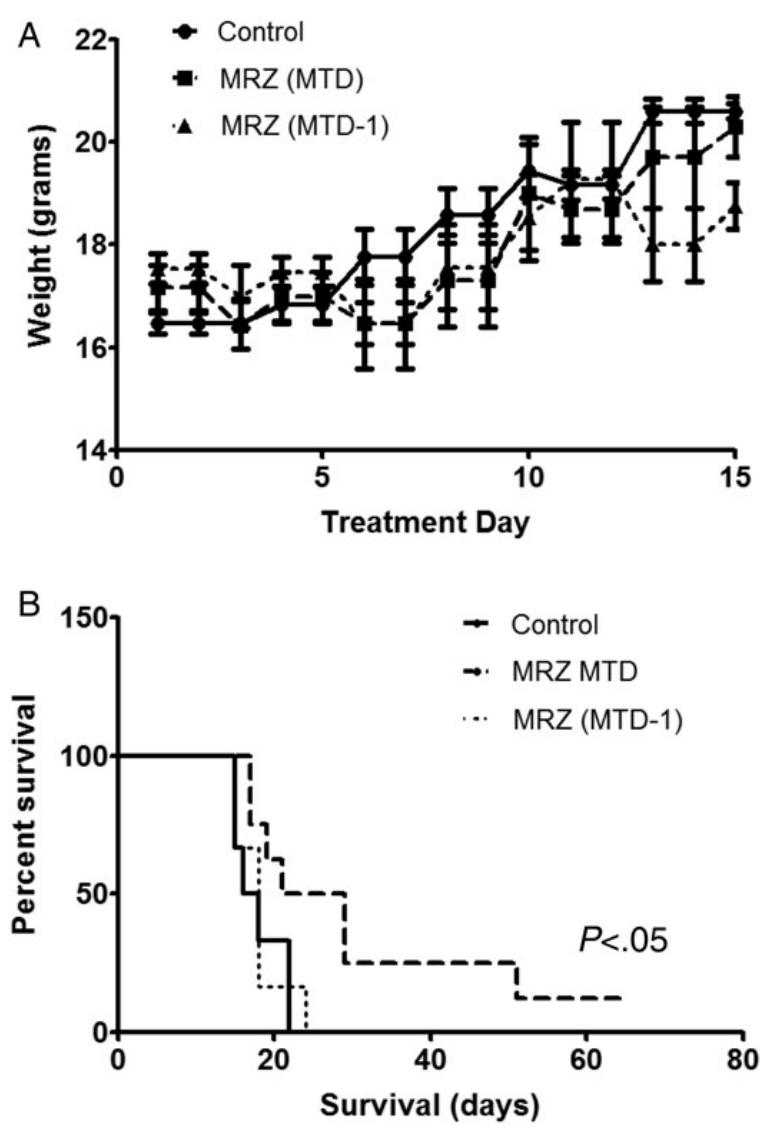

Fig. 5. Marizomib prolonged animal survival in an orthotopic mouse glioma xenograft model. (A) Control vehicle or marizomib was administered twice weekly for 2.5 weeks (on days 1, 4, 8, 11, and 15) into the tail vein of athymic BALB/c nu/nu mice. There was no statistical difference in the weight of animals among the vehicle control, maximum tolerated dose group (MTD: $200 \mu \mathrm{g} / \mathrm{kg}$ ) and lower dose group (MTD-1: $150 \mu \mathrm{g} / \mathrm{kg}$ ). (B) Kaplan-Meier survival probability plots of tumor-bearing mice in vehicle or marizomib treatment groups $(n=$ $6-8$ ), using the log-rank method to test for a difference between groups.

progressing through phase $1 / 2$ clinical trials for multiple myeloma at leading clinical sites in the US and Australia (http://www. clinicaltrials.gov, NCT02103335). Our group is currently embarking on a phase 1 study of marizomib in recurrent GBM patients (NCT02330562). Additional research into the use of marizomib as a potential treatment for malignant gliomas is clearly warranted.

\section{Supplementary Material}

Supplementary material is available at Neuro-Oncology Journal online (http://neuro-oncology.oxfordjournals.org/).

\section{Funding}

This study was supported in part by donations from the Ralph and Suzanne Stern family, and the National Cancer Institute of the National
Institutes of Health under Award Number P30CA062203. Triphase Accelerator Corporation provided additional support for animal studies.

\section{Acknowledgments}

We acknowledge Shadi Safai, Yen Vuong, Sudha Warrier, and Xing Gong for their technical assistance.

Conflict of interest statement. G.K.L., A.M., F.J.B., and M.T. are employees of Triphase Accelerator Corporation, the company that manufactures marizomib. A.D. and D.A.B. are principal investigators of a phase 1 clinical trial of marizomib in recurrent GBM patients (NCT02330562). The remaining authors declare no potential conflict of interest.

\section{References}

1. Ostrom QT, Gittleman H, Liao P, et al. CBTRUS statistical report: primary brain and central nervous system tumors diagnosed in the United States in 2007-2011. Neuro Oncol. 2014;16(suppl 4): iv1-iv63.

2. Friedman HS, Kerby T, Calvert H. Temozolomide and treatment of malignant glioma. Clin Cancer Res. 2000;6(7):2585-2597.

3. Hegi ME, Diserens AC, Godard S, et al. Clinical trial substantiates the predictive value of 0-6-methylguanine-DNA methyltransferase promoter methylation in glioblastoma patients treated with temozolomide. Clin Cancer Res. 2004;10(6):1871-1874.

4. Bredel M, Zentner J. Brain-tumour drug resistance: the bare essentials. Lancet Oncol. 2002;3:397-406.

5. Gong X, Schwartz PH, Linskey ME, Bota DA. Neural stem/progenitors and glioma stem-like cells have differential sensitivity to chemotherapy. Neurology. 2011;76(13):1126-1134.

6. Bao S, Wu Q, Sathornsumetee $S$, et al. Stem cell-like glioma cells promote tumor angiogenesis through vascular endothelial growth factor. Cancer Res. 2006;66(16):7843-7848.

7. Bao $\mathrm{S}, \mathrm{Wu} \mathrm{Q}, \mathrm{McLendon} \mathrm{RE}$, et al. Glioma stem cells promote radioresistance by preferential activation of the DNA damage response. Nature. 2006;444(7120):756-760.

8. Liu G, Yuan X, Zeng Z, et al. Analysis of gene expression and chemoresistance of CD133+ cancer stem cells in glioblastoma. Mol Cancer. 2006;5(1):67.

9. Cai J, Cheng A, Luo Y, et al. Membrane properties of rat embryonic multipotent neural stem cells. J Neurochem. 2004;88:212-226.

10. Ciechanover A, Orian A, Schwartz AL. The ubiquitin-mediated proteolytic pathway: mode of action and clinical implications. J Cell Biochem Suppl. 2000;34:40-51.

11. Adams J, Palombella VJ, Elliott PJ. Proteasome inhibition: a new strategy in cancer treatment. Invest New Drugs. 2000;18(2): 109-121.

12. McConkey DJ, Zhu K. Mechanisms of proteasome inhibitor action and resistance in cancer. Drug Resist Updat. 2008;11(4-5): 164-179.

13. Crawford LJ, Walker B, Irvine AE. Proteasome inhibitors in cancer therapy. J Cell Commun Signal. 2011;5(2):101-110.

14. Yang Y, Yu X. Regulation of apoptosis: the ubiquitous way. Faseb $\mathrm{J}$. 2003;17(8):790-799.

15. Chauhan D, Hideshima T, Anderson KC. Targeting proteasomes as therapy in multiple myeloma. Adv Exp Med Biol. 2008;615: $251-260$. 
16. LeBlanc R, Catley LP, Hideshima T, et al. Proteasome inhibitor PS-341 inhibits human myeloma cell growth in vivo and prolongs survival in a murine model. Cancer Res. 2002;62(17): 4996-5000.

17. Yin D, Zhou H, Kumagai T, et al. Proteasome inhibitor PS-341 causes cell growth arrest and apoptosis in human glioblastoma multiforme (GBM). Oncogene. 2005;24(3):344-354.

18. Yu J, Tiwari S, Steiner P, Zhang L. Differential apoptotic response to the proteasome inhibitor Bortezomib [VELCADE, PS-341] in Bax-deficient and p21-deficient colon cancer cells. Cancer Biol Ther. 2003;2(6):694-699.

19. Yu LJ, Riordan B, Hatsis $P$, et al. Study of brain and whole blood PK/PD of bortezomib (VELCADE $\left.{ }^{\circledR}\right)$ in rat models. J Clin Oncol. 2006;24(suppl 18):12036.

20. Labussiere M, Pinel S, Delfortrie S, Plenat F, Chastagner P. Proteasome inhibition by bortezomib does not translate into efficacy on two malignant glioma xenografts. Oncol Rep. 2008; 20(5):1283-1287.

21. Phuphanich S, Supko JG, Carson K, et al. Phase I trial of bortezomib in adults with recurrent malignant glioma. J Clin Oncol. 2006; 24(suppl 18):1567.

22. Miller CP, Ban K, Dujka ME, et al. NPI-0052, a novel proteasome inhibitor, induces caspase-8 and ROS-dependent apoptosis alone and in combination with HDAC inhibitors in leukemia cells. Blood. 2007;110(1):267-277.

23. Miller CP, Manton CA, Hale R, et al. Specific and prolonged proteasome inhibition dictates apoptosis induction by marizomib and its analogs. Chem Biol Interact. 2011;194(1):58-68.

24. Vlashi E, Mattes M, Lagadec C, et al. Differential Effects of the Proteasome Inhibitor NPI-0052 against Glioma Cells. Transl Oncol. 2010;3(1):50-55.

25. Schwartz PH, Bryant PJ, Fuja TJ, Su H, O'Dowd DK, Klassen H. Isolation and characterization of neural progenitor cells from post-mortem human cortex. J Neurosci Res. 2003;74(6):838-851.

26. Di K, Linskey ME, Bota DA. TRIM11 is overexpressed in high-grade gliomas and promotes proliferation, invasion, migration and glial tumor growth. Oncogene. 2013;32(42):5038-5047.

27. Di K, Keir ST, Alexandru-Abrams D, et al. Profiling Hsp90 differential expression and the molecular effects of the Hsp90 inhibitor IPI-504 in high-grade glioma models. J Neurooncol. 2014; 120(3):473-481.
28. Pistollato F, Chen HL, Rood BR, et al. Hypoxia and HIF1alpha repress the differentiative effects of BMPs in high-grade glioma. Stem Cells. 2009;27(1):7-17.

29. Williamson MJ, Blank JL, Bruzzese FJ, et al. Comparison of biochemical and biological effects of ML858 (salinosporamide A) and bortezomib. Mol Cancer Ther. 2006;5(12):3052-3061.

30. Vlashi E, Kim K, Lagadec C, et al. In vivo imaging, tracking, and targeting of cancer stem cells. J Natl Cancer Inst. 2009;101(5): 350-359.

31. Lim DA, Cha S, Mayo MC, et al. Relationship of glioblastoma multiforme to neural stem cell regions predicts invasive and multifocal tumor phenotype. Neuro Oncol. 2007;9(4):424-429.

32. Ling $Y H$, Liebes L, Zou Y, Perez-Soler R. Reactive oxygen species generation and mitochondrial dysfunction in the apoptotic response to Bortezomib, a novel proteasome inhibitor, in human H460 non-small cell lung cancer cells. J Biol Chem. 2003;278(36): 33714-33723.

33. Ruschak AM, Slassi M, Kay LE, Schimmer AD. Novel proteasome inhibitors to overcome bortezomib resistance. J Natl Cancer Inst. 2011;103(13):1007-1017.

34. Ohshima-Hosoyama S, Davare M, Hosoyama T, Nelon L, Keller C. Bortezomib stabilizes NOXA and triggers ROS-associated apoptosis in medulloblastoma. J Neurooncol. 2011;105(3):475-483.

35. Singh AV, Palladino MA, Lloyd GK, Potts BC, Chauhan D, Anderson KC. Pharmacodynamic and efficacy studies of the novel proteasome inhibitor NPI-0052 (marizomib) in a human plasmacytoma xenograft murine model. Br J Haematol. 2010; 149(4):550-559.

36. Cusack JC Jr., Liu R, Xia L, et al. NPI-0052 enhances tumoricidal response to conventional cancer therapy in a colon cancer model. Clin Cancer Res. 2006;12(22):6758-6764.

37. Sloss CM, Wang F, Liu R, et al. Proteasome inhibition activates epidermal growth factor receptor (EGFR) and EGFR-independent mitogenic kinase signaling pathways in pancreatic cancer cells. Clin Cancer Res. 2008;14(16):5116-5123.

38. Vlachostergios PJ, Papandreou CN. Efficacy of low dose temozolomide in combination with bortezomib in U87 glioma cells: a flow cytometric analysis. Arch Med Sci. 2015;11(2):307-310.

39. Lin L, Gaut D, Hu K, Yan H, Yin D, Koeffler HP. Dual targeting of glioblastoma multiforme with a proteasome inhibitor (Velcade) and a phosphatidylinositol 3-kinase inhibitor (ZSTK474). Int $J$ Oncol. 2014;44(2):557-562. 